This item was submitted to Loughborough's Research Repository by the author.

Items in Figshare are protected by copyright, with all rights reserved, unless otherwise indicated.

\title{
Nonlinear backreaction in a quantum mechanical SQUID
}

PLEASE CITE THE PUBLISHED VERSION

http://dx.doi.org/10.1103/PhysRevB.64.180504

\section{PUBLISHER}

(C) American Physical Society

VERSION

VoR (Version of Record)

LICENCE

CC BY-NC-ND 4.0

\section{REPOSITORY RECORD}

Ralph, J.F., T.D. Clark, Mark J. Everitt, and P.B. Stiffell. 2019. "Nonlinear Backreaction in a Quantum Mechanical SQUID”. figshare. https://hdl.handle.net/2134/12086. 
This item was submitted to Loughborough's Institutional Repository (https://dspace.lboro.ac.uk/) by the author and is made available under the following Creative Commons Licence conditions.

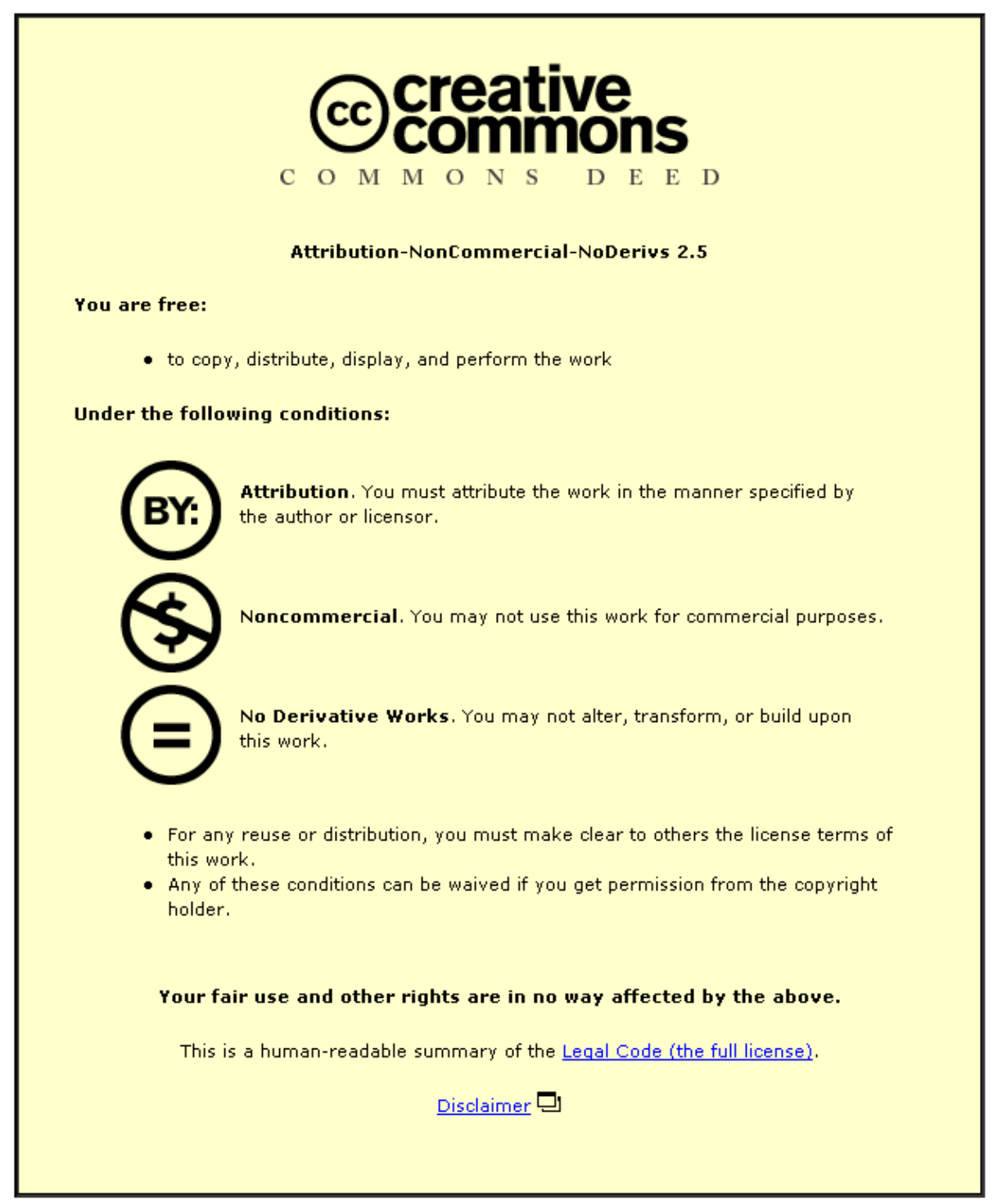

For the full text of this licence, please go to: http://creativecommons.org/licenses/by-nc-nd/2.5/ 


\title{
Nonlinear backreaction in a quantum mechanical SQUID
}

\author{
J. F. Ralph, ${ }^{1, *}$ T. D. Clark, ${ }^{2}$ M. J. Everitt, ${ }^{2}$ and P. Stiffell ${ }^{2}$ \\ ${ }^{1}$ Department of Electrical Engineering and Electronics, The University of Liverpool, Brownlow Hill, \\ Liverpool, L69 3GJ, United Kingdom \\ ${ }^{2}$ School of Engineering, The University of Sussex, Falmer, Brighton, BN1 9QT, United Kingdom
}

(Received 19 June 2001; published 3 October 2001)

\begin{abstract}
In this paper we discuss the coupling between a quantum mechanical superconducting quantum interference device (SQUID) and an applied static magnetic field. We demonstrate that the backreaction of a SQUID on the applied field can interfere with the ability to bias the SQUID at values of the static (dc) magnetic flux at, or near to, transitions in the quantum mechanical SQUID.
\end{abstract}

DOI: 10.1103/PhysRevB.64.180504

PACS number(s): 74.50. $+\mathrm{r}, 03.65 .-\mathrm{w}$, 85.25.Dq

\section{INTRODUCTION}

There are a number of systems that are currently being considered as candidates for the construction of qubits, quantum logic gates, and quantum computers. ${ }^{1}$ Some of the systems, notably atoms in magnetic traps ${ }^{2}$ and nuclear magnetic resonance (NMR) systems, ${ }^{3}$ have had some success in performing the elementary operations that would be required in a large scale quantum computer. These systems benefit from the relatively weak coupling between the quantum degrees of freedom used for the qubits and the external environment. This can result in coherence times that are fairly long compared to the timescales used in the quantum calculations. However, these systems are not necessarily seen as viable technologies for quantum computing in the longer term. The difficulties involved in constructing large scale quantum circuits using such systems are likely to be a limiting factor. A more realistic solution would be to develop qubit systems using solid state systems that would allow systems to be fabricated easily and repeatedly. The recent demonstration of macroscopic coherence in a superconducting quantum interference device (SQUID) ring ${ }^{4,5}$ (consisting of a thick superconducting ring containing one or more Josephson weak link devices) has added significant weight to the idea of using SQUID's in quantum logic systems, ${ }^{6-10}$ although other technologies are also being actively considered. ${ }^{1,9}$

In this paper, we consider one aspect of the quantum mechanical SQUID that has previously been overlooked, and we discuss how it may influence the construction and design of quantum logic gates based on SQUID devices. The subject of this paper is the effect that the SQUID has on an applied magnetic field. Previous work has concentrated on the appearance of nonlinear behavior in SQUID systems when they are coupled to radio-frequency oscillator circuits ("tank" circuits). This system has been investigated in both the classical $^{11,12}$ and the quantum regimes, ${ }^{13,14}$ and has been shown to contain a range of interesting nonlinear effects. In the current work, we concentrate on a more fundamental problem: the nonlinear effect of the SQUID on a static magnetic flux. In particular, we look at problems associated with fixing the classical magnetic flux bias for a quantum mechanical SQUID at, or near, a quantum mechanical transition or resonance.

We present results that suggest that the backreaction of the SQUID on the static magnetic field can alter the apparent shape of the quantum mechanical resonance even when the coupling between the field and the SQUID ring is weak. These results are important for quantum logic gates constructed using SQUID's because it is through a static (dc) magnetic flux that the behavior of a SQUID qubit is controlled. $^{6-10}$ There are some differences between the way in which the flux bias is used, but the effect of the backreaction should remain where a quantum resonance is being excited by an external time-varying field, although the size of the effect will vary from system to system. This is particularly relevant for $\pi$-SQUID's, where the adjacent wells in magnetic flux are degenerate at zero applied flux, ${ }^{10}$ which may reduce the significance of the effect.

\section{TRANSITIONS IN A QUANTUM MECHANICAL SQUID RING}

The behavior of a quantum mechanical SQUID ring in the presence of a time-dependent field is given by the timedependent Schrödinger equation (TDSE), which can be solved using perturbative methods ${ }^{4}$ or nonperturbative methods. ${ }^{15,16}$ In the latter case, complex multiphoton transitions can be found for both semiclassical ${ }^{15}$ and fully quantum mechanical descriptions of the applied field. ${ }^{16}$ We adopt the nonperturbative, semiclassical approach described in Ref. 15 , although we will restrict ourselves to single-photon, perturbative transitions for simplicity. However, the nonlinear analysis presented below is applicable to the nonperturbative, multiphoton transitions and to the transitions predicted using perturbative methods. In the case of the multiphoton transitions, the complexity of the transitions would make it difficult to separate the nonlinear effects from the transitions. Perturbative methods do provide an indication of the occurrence of a transition and an estimate of the linewidth of that transition, but they do not allow the shape of the resonance to be calculated, which is crucial for the determination of the nonlinear backreaction.

A thick superconducting ring containing a single weak link ring [a radio-frequency (rf-)SQUID ring] is often described in terms of a single macroscopic degree of freedom, $\Phi_{s}$, corresponding to the enclosed magnetic flux, with the electric displacement flux $Q_{s}$ playing the role of the conjugate momentum (strictly speaking the conjugate momentum 


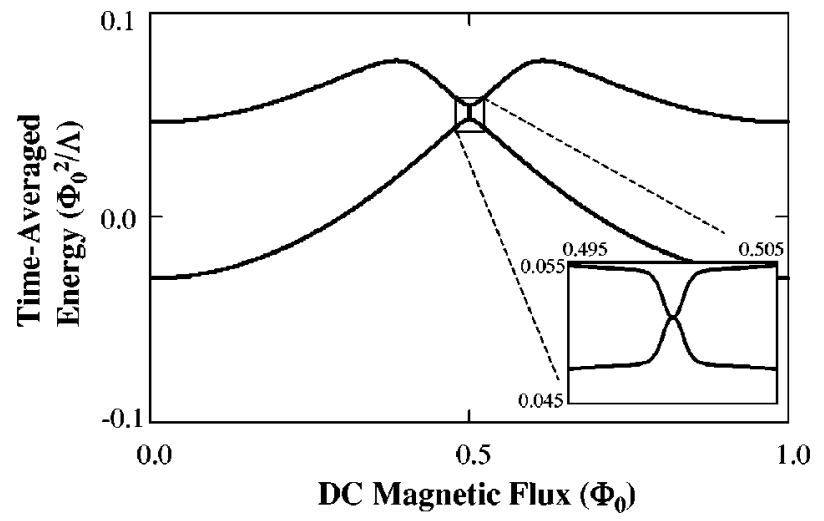

FIG. 1. Time-averaged energy levels for the first two energy states of an rf-SQUID ring, showing a perturbative, single-photon transition at $\Phi_{d c}=0.5 \Phi_{0}$. The inset shows the same transition in more detail. (The system parameters are given in the text.)

is $-Q_{s}$, the commutator being given by $\left.\left[Q_{s}, \Phi_{s}\right]=i \hbar\right)$. The Hamiltonian for the ring is given by

$$
H_{s}\left(\Phi_{x}(t)\right)=\frac{Q_{s}^{2}}{2 C_{s}}+\frac{\left(\Phi_{s}-\Phi_{x}(t)\right)^{2}}{2 \Lambda}-\hbar \nu \cos \left(\frac{2 \pi \Phi_{s}}{\Phi_{0}}\right)
$$

where $C_{s}$ is the effective capacitance of the weak link, $\Lambda$ is the inductance of the ring, $\nu$ is the (angular) tunneling frequency of the weak link (related to the critical current $I_{c}$ by $\nu=I_{c} / 2 e$, where $2 e$ is the charge of an electron pair), $\Phi_{0}$ $=h / 2 e=2 \times 10^{-15} \mathrm{~Wb}$ is the magnetic flux quantum, and $\Phi_{x}(t)$ is the external magnetic flux applied to the ring. In this paper, we assume that the magnetic flux contains a timedependent term to drive the resonance [typically at microwave frequencies $\Phi_{m w}(t)=\Phi_{m w}(t) \sin \left(\omega_{m w} t+\delta\right)$ where $\delta$ is an arbitrary phase], and a static dc magnetic flux to provide the bias point $\Phi_{d c}$. In experiments, such as those described in Refs. 4, 5, and 17, the microwaves are usually introduced via a coaxial cable acting as a transmission line, and the dc flux is applied by inductively coupling a current-carrying coil to the SQUID.

Figure 1 shows the first two (time-averaged) energy levels corresponding to the SQUID ground state and first excited state for the SQUID ring with inductance $\Lambda=3 \times 10^{-10} \mathrm{H}$, weak link capacitance $C_{s}=1 \times 10^{-16} \mathrm{~F}, \hbar \nu=0.07 \Phi_{0}^{2} / \Lambda$ and subject to a microwave field of frequency $f_{m w}=\omega_{m w} / 2 \pi$ $=144.7229 \mathrm{GHz}$ and amplitude $\Phi_{m w}=5 \times 10^{-5} \Phi_{0}$, where the time-averaged energies are defined by

$$
\left\langle\left\langle E\left(\Phi_{d c}\right)\right\rangle\right\rangle_{\kappa}=\frac{\omega_{m w}}{2 \pi} \int_{0}^{2 \pi / \omega_{m w}} d t\left\langle E\left(\Phi_{x}(t)\right)\right\rangle_{\kappa}
$$

and $\left\langle E\left(\Phi_{x}(t)\right)\right\rangle_{\kappa}$ is the instantaneous energy eigenvalue of the $\kappa$ th instantaneous energy state of the Hamiltonian (1), as described in Ref. 15, and correspond to the Floquet quasienergies that are used extensively in quantum optics. ${ }^{18}$ For systems where the quantum transitions are at microwave frequencies, it is assumed that it is the time-averaged energies (or the corresponding time-averaged screening currents, see below) that induce a nonlinear backreaction in the dc coil.
This approximation is based on the assumption that the fluctuations in the dc magnetic flux occur at frequencies much lower than those present in the SQUID or the applied microwaves.

\section{THE EFFECT OF THE BACKREACTION ON THE dc BIAS}

The time-averaged energies shown in Fig. 1 are calculated assuming that the dc bias can be set at a desired value with an arbitrary accuracy. At first glance one might assume that, in a real system, there will be some noise that will tend to 'blur' out any very small features but, as long as the magnetic flux noise is small compared to the size of the features, the general form should be preserved. However, this is based on the assumption that it is the static magnetic flux that is the control parameter. In practice, it is not the dc magnetic flux that is set, rather it is the dc current that flows through the coil that is fixed. The current flowing in the dc coil induces a flux that couples to the SQUID, which induces a screening current in the SQUID ring that couples back to the dc coil modifying the true value of the applied dc flux. This backreaction effect in quantum mechanical SQUID rings has been studied in the context of an rf-oscillator/SQUID system, where it is possible to derive an equation of motion for a classical oscillator in the presence of an rf-SQUID that includes the effect of coupling to all orders. ${ }^{13,14}$ We model the dc coil as an oscillator (for the moment at least), and use the definition of the mutual inductance $M$ between a SQUID and an external inductive circuit,

$$
\begin{aligned}
& \Phi_{s}=\Lambda I_{s}+M I_{t}, \\
& \Phi_{t}=L_{t} I_{t}+M I_{s},
\end{aligned}
$$

where $\Phi_{t}$ is the magnetic flux in the oscillator, and the oscillator is characterized by a capacitance $C_{t}$, and inductance $L_{t}$. The Hamiltonian for the combined system can be written in the form, ${ }^{13}$

$$
\begin{aligned}
H= & \frac{Q_{t}^{2}}{2 C_{t}}+\frac{\Phi_{t}^{2}}{2 L_{t}\left(1-K^{2}\right)}-\Phi_{t} I_{i n}+\frac{K^{2} \Phi_{t} \Phi_{s}}{M\left(1-K^{2}\right)}+\frac{Q_{s}^{2}}{2 C_{s}} \\
& +\frac{\Phi_{s}^{2}}{2 \Lambda\left(1-K^{2}\right)}-\hbar \nu \cos \left(\frac{2 \pi \Phi_{s}}{\Phi_{0}}\right) \\
= & \frac{Q_{t}^{2}}{2 C_{t}}+\frac{\Phi_{t}^{2}}{2 L_{t}}-\Phi_{t} I_{i n} \\
& +\frac{Q_{s}^{2}}{2 C_{s}}+\frac{\left(\Phi_{s}-\mu \Phi_{t}\right)^{2}}{2 \Lambda\left(1-K^{2}\right)}-\hbar \nu \cos \left(\frac{2 \pi \Phi_{s}}{\Phi_{0}}\right) \\
= & H_{t}\left(I_{i n}\right)+H_{s}\left(\mu \Phi_{t}\right),
\end{aligned}
$$

where $I_{\text {in }}$ is the external current applied to the oscillator and the coupling coefficients are given by $K^{2}=M^{2} / \Lambda L_{t}$ and $\mu$ $=M / L_{t}$. From the Hamiltonian (5), we can see that the effect of the coupling on the SQUID can be represented as shifting the effective inductance of the SQUID ring by a factor $\left(1-K^{2}\right), \Lambda \rightarrow \Lambda\left(1-K^{2}\right)$.

Averaging over the quantum behavior, it is then possible to derive a classical equation of motion for the magnetic flux in the oscillator coil as a function of the applied current, 


$$
C_{t} \frac{d^{2} \Phi_{t}}{d t^{2}}+\frac{1}{R_{t}} \frac{d \Phi_{t}}{d t}+\frac{\Phi_{t}}{L_{t}}=I_{i n}+\frac{\mu\left\langle\left\langle I_{S}\left(\mu \Phi_{t}\right)\right\rangle\right\rangle_{\kappa}}{\left(1-K^{2}\right)},
$$

where we have inserted a resistance $R_{t}$, and the timeaveraged screening current in the SQUID ring is calculated using the bare (unrenormalized) inductance of the SQUID ring $\Lambda$, leading to the $1 /\left(1-K^{2}\right)$ factor in the last term. (In Ref. 13 the average screening current in the ring is calculated using the value of the renormalized inductance, which removes the multiplicative factor, but does not change the behavior predicted by the equation.) The inclusion of the timeaveraged screening current,

$$
\left\langle\left\langle I_{S}\left(\Phi_{d c}\right)\right\rangle\right\rangle_{\kappa}=-\frac{\left\langle\left\langle\Phi_{s}\left(\Phi_{d c}\right)\right\rangle\right\rangle_{\kappa}}{\Lambda}=-\frac{\partial\left\langle\left\langle E\left(\Phi_{d c}\right)\right\rangle\right\rangle_{\kappa}}{\partial \Phi_{d c}},
$$

is equivalent to the use of the Born-Oppenheimer approximation $^{13,19}$ that is used in atomic and molecular calculations for systems that vary over very different time scales (e.g., it is used to separate the slow dynamics of nuclei from the very fast dynamics of electrons). In this situation, the Born-Oppenheimer approximation is used to separate the dynamics of the SQUID/microwave system from the dynamics associated with the dc coil.

Since the subject of this paper is the behavior of the dc magnetic flux coil and all fluctuations associated with the dc coil are assumed to have a very low frequency, we can approximate the oscillator equation (6) by

$$
\frac{\Phi_{d c}}{L_{d c}}=I_{d c}+\frac{\mu\left\langle\left\langle I_{S}\left(\mu \Phi_{d c}\right)\right\rangle\right\rangle_{\kappa}}{\left(1-K^{2}\right)},
$$

where the quantities now relate to the dc coil and the dc current that is applied to it. This is the equation that we can use to determine how much static flux couples to the SQUID ring. It is nonlinear, so that the dc flux is not necessarily proportional to the applied current. For a fixed current level we can solve this equation to find the dc flux level that couples to the SQUID. Given a range of these dc values, it is possible to use the calculated, time-averaged energy levels to predict the apparent energy level structure. Figure 2 shows the time-averaged energy levels from the inset of Fig. 1 as a function of the applied dc current for several different coupling strengths, including the original energies for comparison.

The three examples given in Fig. 2 show very different behavior. The first, Fig. 2(a) with $K^{2}=0.01$ corresponding to $\mu=0.055$, shows that the width of the resonance in the ground state is reduced by the effect of the backreaction, while the first excited state is broadened. Of course, it would normally be much easier to see the ground state behavior than the excited states because of environmental effects, but we include both for completeness. In Fig. 2(b), $K^{2}=0.03$ and $\mu=0.095$, a hysteresis loop appears on either side of the resonance. This is because there are multiple solutions to Eq. (8), leading to multiple allowable flux values for a single value of the dc current. The situation is even more extreme in Fig. 2(c), $K^{2}=0.05$ and $\mu=0.122$, where the nonlinearities (a)

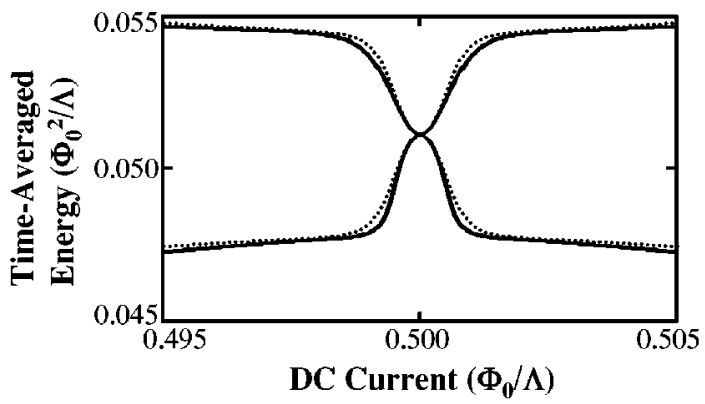

(b)

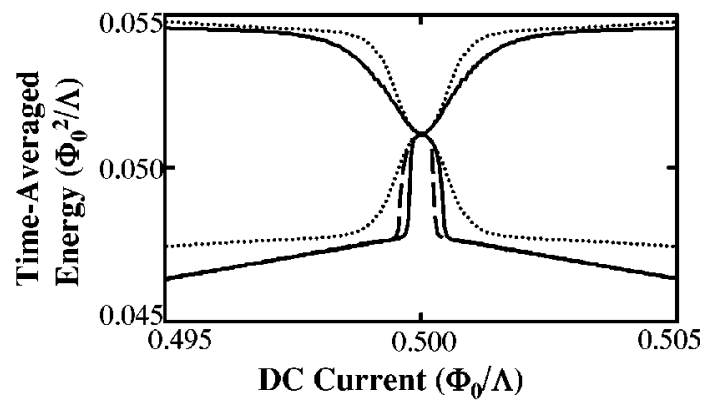

(c)

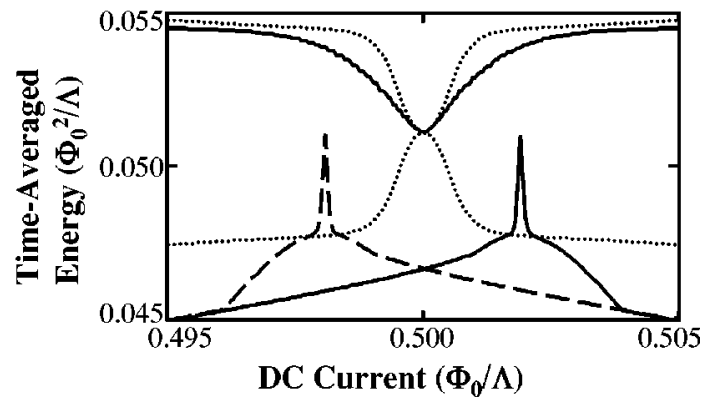

FIG. 2. Time-averaged energy levels as a function of applied dc current for a dc coil with $L_{d c}=1 \times 10^{-9} \mathrm{H}$ : (a) $K^{2}=0.01$ and $\mu$ $=0.055$, (b) $K^{2}=0.03$ and $\mu=0.095$, (c) $K^{2}=0.05$ and $\mu=0.122$. The original energies are shown as a dotted line for comparison. In (b) and (c) increasing current is shown as a solid line and decreasing current is shown as a dashed line.

are now so severe that the ground state resonance no longer looks anything like the original. The single resonance shown in the inset of Fig. 1 has been split, giving asymmetric resonances that depend on whether the dc current is being ramped (quasistatically) up or down. In each of these figures, the first excited state behavior is relatively consistent. This is because the effect of the backreaction is to broaden the features, reducing the effect of the nonlinearity rather than narrowing the resonance, which increase the apparent size of the screening current [which is related to the derivative of the energy level via Eq. (7)]. It should be stressed that the behavior shown in Fig. 2 is not dependent on the particular solution of the time-dependent Schrödinger equation chosen for this paper. The same type of behavior should be seen in the region of any quantum transition or resonance with the same general shape as that shown in the inset of Fig. 1. Equally, if the time-dependent field is removed the effect of the nonlinear backreaction will be very much reduced because it is dependent on the curvature of the time-averaged energy in the SQUID ring. When the time-dependent field is 
applied, the effect of the nonlinearity is much stronger because of the "sharp" shape of the resonance compared to the background curvature of the time-independent energy levels (see Fig. 1).

Although this is an interesting nonlinear effect in its own right, the importance of the backreaction is mainly in what it tells us about the ability to control or bias a quantum mechanical SQUID at or near to one of its transitions. Near to a transition, the (time-averaged) screening currents generated by the ring are very nonlinear and can generate very strong nonlinear behavior, such as hysteresis. If one were to try to hold the system near to one of these regions of strongly nonlinear behavior, the dc flux might not behave in a predictable manner when subject to small amounts of noise, with the system "hopping" around between the different possible flux states. This could cause problems if the system was required to operate in one of these regimes to create quantum entanglements between elements in a quantum circuit. The only way to reduce these effects is to reduce the coupling between the dc coil and the SQUID ring. (The behavior shown in Fig. 2 is only weakly dependent on the individual inductances for the SQUID and the dc coil.) Although it may be possible to reduce the couplings for individual SQUID systems, there may be practical limitations to this approach when designing large scale systems with many SQUID devices, of the type required for a large scale quantum computer based on SQUID technology. If the coupling is reduced between a SQUID and its dc coil, to reduce the effects of the backreaction, it becomes difficult to decouple the dc coil from the neighboring SQUID's, which may introduce problems with cross coupling between qubits and/or additional unwanted environmental effects.

\section{CONCLUSIONS}

The subject of this paper has been the control of quantum mechanical SQUID rings at, or near to, a transition using an external static magnetic flux. We have shown that the effect of the backreaction of the SQUID on the dc coil can be significant near to a transition, even when the coupling between the two systems is weak. For the example used in this paper the effect is significant even when the coupling is around $1 \%\left(K^{2}=0.01\right)$. The appearance of nonlinear behavior in the dc coil, such as multiple stable solutions and hysteresis, could lead to unpredictable behavior in the SQUID and disturb the correct operation of a qubit/quantum gate based on SQUID devices.

\section{ACKNOWLEDGMENTS}

The authors would like to thank the United Kingdom National Endowment for Science Technology and the Arts (NESTA) and the Engineering and Physical Science Research Council (EPSRC) Quantum Circuits Network for their generous support.
*Email address: jfralph@liv.ac.uk

${ }^{1}$ D. P. DiVincenzo, in Mesoscopic Electron Transport, edited by L. L. Sohn, L. P. Kouwenhoven, and G. Schoen, Vol. 345 of NATO ASI Series E (Kluwer, Dordrecht, 1997), p. 657; D. P. DiVincenzo and D. Loss, Superlattices Microstruct. 23, 419 (1998); J. Magn. Magn. Mater. 200, 202 (1999).

${ }^{2}$ C. Monroe, D. M. Meekhof, B. E. King, W. M. Itano, and D. J. Wineland, Phys. Rev. Lett. 75, 4714 (1995).

${ }^{3}$ N. Gershenfeld and I. Cheng, Science 275, 350 (1997); D. Cory, A. Fahmy, and T. Havel, Proc. Natl. Acad. Sci. U.S.A. 94, 1634 (1997).

${ }^{4}$ J. R. Friedman, V. Patel, W. Chen, S. K. Tolpygo, and J. E. Lukens, Nature (London) 406, 43 (2000).

${ }^{5}$ C. H. van der Wal, A. C. J. ter Haar, F. K. Wilhem, R. N. Schouten, C. J. P. M. Harmans, T. P. Orlando, S. Lloyd, and J. E. Mooij, Science 290, 773 (2000).

${ }^{6}$ M. F. Bocko, A. M. Herr, and M. J. Feldman, IEEE Trans. Appl. Supercond. 7, 3638 (1997).

${ }^{7}$ T. P. Orlando, J. E. Mooji, L. Tian, C. H. van der Wal, L. S. Levitov, S. Lloyd, and J. J. Mazo, Phys. Rev. B 60, 15398 (1999).

${ }^{8}$ F. Chiarello, Phys. Lett. A 277, 189 (2000).

${ }^{9}$ T. P. Spiller, Fortschr. Phys. 48, 1075 (2000).

${ }^{10}$ R. R. Schulz, B. Chesca, B. Goetz, C. W. Schneider, A. Schmehl,
H. Bielefeldt, H. Hilgenkamp, and J. Mannhart, Appl. Phys. Lett. 76, 912 (2000).

${ }^{11}$ M. P. Soerensen, M. Barchelli, P. L. Christinasen, and A. R. Bishop, Phys. Lett. 109A, 347 (1985).

${ }^{12}$ W. C. Schieve, A. R. Bulsara, and E. W. Jacobs, Phys. Rev. A 37, 3541 (1988)

${ }^{13}$ J. F. Ralph, T. P. Spiller, T. D. Clark, R. J. Prance, and H. Prance, Int. J. Mod. Phys. B 8, 2637 (1994).

${ }^{14}$ J. Diggins, J. F. Ralph, T. P. Spiller, T. D. Clark, H. Prance, and R. J. Prance, Phys. Rev. E 49, 1854 (1994); T. D. Clark, J. F. Ralph, R. J. Prance, H. Prance, J. Diggins, and R. Whiteman, ibid. 57, 4035 (1998)

${ }^{15}$ T. D. Clark, J. Diggins, J. F. Ralph, M. J. Everritt, R. J. Prance, H. Prance, and R. Whiteman, Ann. Phys. (N.Y.) 268, 1 (1998).

${ }^{16}$ M. J. Everitt, P. Stiffell, T. D. Clark, A. Vourdas, J. F. Ralph, H. Prance, and R. J. Prance, Phys. Rev. B 63, 144530 (2001).

${ }^{17}$ R. Whiteman, T. D. Clark, R. J. Prance, H. Prance, V. Schllmann, J. F. Ralph, M. Everitt, and J. Diggins, J. Mod. Opt. 45, 1175 (1998).

${ }^{18}$ E.g., C. Cohen-Tannoudji, J. Dupont-Roc, and G. Grynberg, Atom-Photon Interactions (Wiley, New York, 1992), Chaps. III and IV; J. Shirley, Phys. Rev. 138, B979 (1965); S. I. Chu, Adv. At. Mol. Phys. 21, 197 (1985).

${ }^{19}$ M. Born, Z. Phys. 38, 803 (1926). 